\title{
Scaling multimode fibre IM/DD transmission capacity through spatial-spectral multiplexing
}

\author{
C. P. Tsekrekos ${ }^{1}$, N. G. Varithimiadis ${ }^{2}$, D. I. Kassos ${ }^{2}$, D. Syvridis ${ }^{2}$, S. Sygletos ${ }^{1}$ \\ ${ }^{1}$ Aston Institute of Photonic Technologies, School of Engineering and Applied Science, Aston University, UK \\ ${ }^{2}$ Department of Informatics and Telecommunications, National and Kapodistrian University of Athens, Greece \\ e-mail: c.tsekrekos@aston.ac.uk
}

\begin{abstract}
Data centre connections can greatly benefit from parallel transmission channels on one multimode fibre (MMF). Shortwave wavelength division multiplexing (SWDM) achieves parallel transmission through spectral multiplexing. Furthermore, MMFs offer a spatial dimension that should be exploited to increase parallel transmission, albeit in a cost-effective way. In this paper, it is shown that SWDM and spatial multiplexing can be combined in intensity modulation and direct detection MMF transmission systems that use selective offset excitation and mode-selective spatial filtering.
\end{abstract}

Keywords: space division multiplexing, wavelength division multiplexing, multimode fibre, data centre network

\section{INTRODUCTION}

Intra-data-centre optical connections are extensively employing graded-index (GI) multimode fibres (MMFs). Such MMF connections do not normally exceed a 500-m reach and should support the Gbps needs of the massive data centre networks. To achieve transmission of $100 \mathrm{Gbps}$ and beyond, the following approaches have been considered: parallel optics in terms of parallel MMFs and MPO connectors, using advanced modulation formats such as pulse amplitude modulation and discrete multitone, and introducing shortwave wavelength division multiplexing (SWDM) by extending the 850-nm window and employing wideband MMFs [1], [2]. These approaches lead to solutions based on intensity modulation and direct detection (IM/DD). However, it is also the case that the MMF modes can be exploited by applying space division multiplexing (SDM) techniques. This can have significant advantages such as increasing the bit-rate-distance product, or reducing the number of parallel MMFs required. Applying SDM with IM/DD on GI-MMFs is possible and can be realized with selective offset excitation and mode-selective spatial filtering (MSSF) [3], [4].

In this paper, we explore the application of IM/DD SDM with offset excitation and MSSF on 50/125 $\mu \mathrm{m}$ GIMMFs in a SWDM scenario. This scenario would require wavelength-selective optical filters that do not impact the mode power distribution that forms each spatial channel. This requirement can be met by WDM filters based on free-space optics and thin film filters [5]. It remains, though, crucial to investigate the application of SDM in terms of offset excitation and MSSF over the wavelength range proposed for SWDM. It is shown here that this IM/DD SDM approach can apply similarly on each SWDM channel, although the mode volume of the GI-MMF varies significantly within the SWDM wavelength range. Furthermore, the case of two IM/DD SDM channels can be realized without any additional signal processing requirements, presenting a very simple and straightforward scenario.

\section{SPATIAL-SPECTRAL MULTIPLEXING IN GI-MMFs}

Coherent detection and digital signal processing techniques have enabled the use of the propagation modes in few-mode fibres and MMFs to apply SDM and increase the bit-rate per wavelength in a single fibre. However, it is also possible to apply SDM with IM/DD by using selective offset excitation at the input of a GI-MMF and MSSF at the receiving end [3], [4]. Selective excitation with multiple offset beams yields near-field intensity patterns (NFIPs) that overlap. MSSF is an optical way to reduce the overlap by transforming disk-like NFIPs into ring-like ones. To achieve this, MSSF projects the NFIP at the output of a GI-MMF with an imaging system with a numerical aperture (NA) lower than the NA of the GI-MMF.

The principle of MSSF applied with GI-MMFs excited by offset beams has been described elsewhere for a single wavelength [3], [4]. To explore the combined application of SWDM and IM/DD SDM, it is necessary to analyse the NFIPs at the output of a GI-MMF when offset excitation and MSSF apply within the wavelength range proposed for SWDM. The NFIP is important in designing the receiving end of such a system [6]. Four different wavelengths are considered, namely, 850, 880, 910, and $940 \mathrm{~nm}$, in accordance with a four-channel SWDM approach [1], [2]. Over this wavelength range, the mode volume of the GI-MMF changes. In the following, a GI-MMF with a core/cladding diameter of 50/125 $\mu \mathrm{m}$ and a parabolic refractive index profile in the core area is considered. This parabolic approximation is close to the power law profile of OM4 and wideband GI-MMFs [7]. The value of the refractive index in the cladding is given by the Sellmeier equation proposed in Ref. [8], while the NA of the GI-MMF is taken to be 0.2. This GI-MMF supports 342 modes at $850 \mathrm{~nm}$ and 274 modes at $940 \mathrm{~nm}$. The objective of the analysis is to investigate the NFIP of the GI-MMF and the impact of MSSF within the SWDM wavelength range, when offset excitation with a single-mode fibre (SMF) applies. 

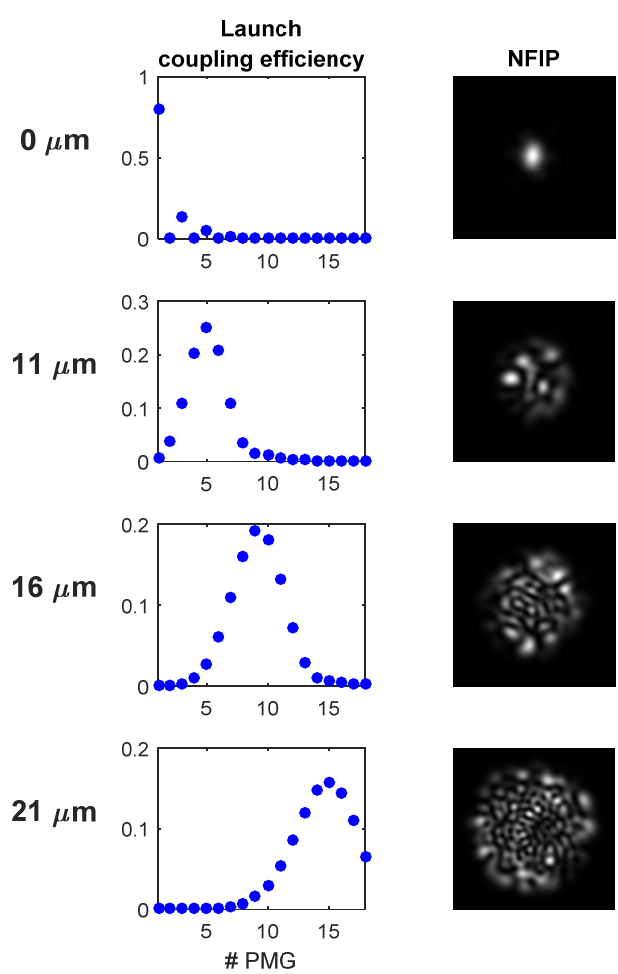
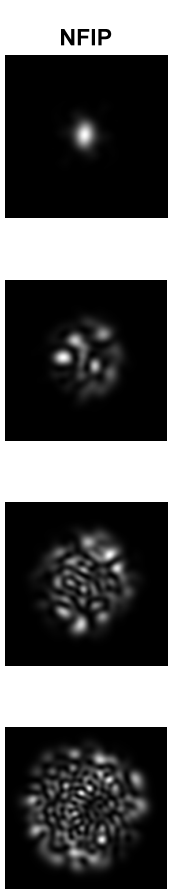
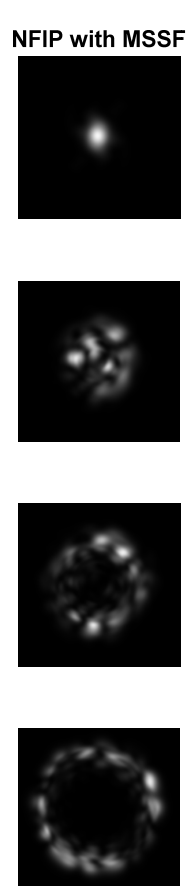
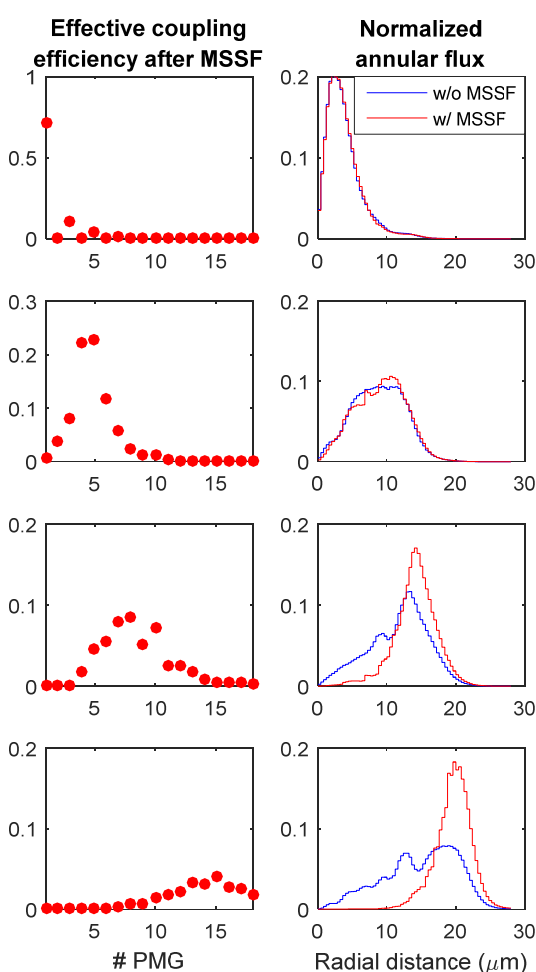

Figure 1. Launch coupling efficiency per principal mode group (PMG), near-field intensity pattern (NFIP) without and with mode-selective spatial filtering (MSSF) after $200 \mathrm{~m}$ of propagation on a 50/125- $\mu \mathrm{m}$ GI-MMF, effective coupling efficiency per PMG that would correspond to the NFIP with MSSF, and normalized annular flux without and with MSSF. Each row corresponds to a different offset launch $(0,11,16$, and $21 \mu \mathrm{m})$. The wavelength is $850 \mathrm{~nm}$ and full intra-PMG mixing is considered.

To analyse the NFIP of the GI-MMF under offset excitation the following approach is used. The GI-MMF mode fields and propagation coefficients are obtained with a full-vectorial finite element method. The field profile used for offset excitation is the mode of a SMF. No angular tilt is introduced between the SMF and the GI-MMF, i.e. their axes are parallel. The SMF is a step-index fibre with a core/cladding diameter of 5.4/125 $\mu \mathrm{m}$. The SMF cladding refractive index is given by the same Sellmeier equation used for the cladding region of the GI-MMF and the NA of the SMF is taken to be 0.1. MSSF is achieved by considering projection of the NFIP at the output of the GI-MMF with an imaging system of $0.1 \mathrm{NA}$ and a magnification factor equal to 1 . The GIMMF propagation model includes differential mode attenuation (DMA), estimated by an empirical formula that uses a modified Bessel function of the first kind [9]. An intrinsic fibre attenuation of $2 \mathrm{~dB} / \mathrm{km}$ (lower-order modes) is used, while the highest-order modes have an attenuation of about $5 \mathrm{~dB} / \mathrm{km}$ (with these values holding, as an approximation, for the four considered wavelengths). Furthermore, a purely monochromatic source is assumed (zero linewidth), which represents the worst-case scenario in terms of the speckle contrast of the NFIP.

In MMFs, apart from the degenerate modes that form the well-known linearly-polarized modes, principal mode groups (PMGs) exist, which are groups of modes with approximately the same propagation coefficient. The GIMMF under investigation has 18 (17) PMGs at 850 and $880 \mathrm{~nm}$ (910 and $940 \mathrm{~nm})$. The mode-field diameter (MFD) of the SMF used is about $60 \%$ of the MFD of the fundamental mode (HE11) of the GI-MMF. This choice is a practical compromise for exciting nearly as few PMGs as possible for different offset positions [10]. The MFD of the SMF is around $6.9 \mu \mathrm{m}(7.6 \mu \mathrm{m})$ at $850 \mathrm{~nm}(940 \mathrm{~nm})$, while the MFD of the HE11 mode of the GI-MMF is around $11.6 \mu \mathrm{m}(12 \mu \mathrm{m})$ at $850 \mathrm{~nm}(940 \mathrm{~nm})$. During propagation mode mixing occurs. This mixing is strongest among modes of the same PMG. Full mixing within a PMG (intra-PMG mixing) is modelled by equally distributing the coupled optical power among the modes of a PMG and taking a mean value of the propagation coefficient for the modes of a PMG [11].

Four radially offset positions are used, in particular, $0,11,16$, and $21 \mu \mathrm{m}$, the values indicating the distance of the SMF axis from the GI-MMF axis. At $850 \mathrm{~nm}$ wavelength, the results are shown in Fig. 1. Each row of Fig. 1 corresponds to an offset position of the launched beam (the mode of the SMF). The first column shows the coupling efficiency per PMG at the input of the GI-MMF, calculated by taking the overlap integrals between the launched SMF mode and each mode of a PMG. For every offset position, the summation of these coupling efficiencies gives the total coupling efficiency of the launched field (coupling loss due to reflections at the MMF input facet is not taken into account). For offsets 0,11 , and $16 \mu \mathrm{m}$, the total coupling efficiency is $100 \%$, and for the largest offset of $21 \mu \mathrm{m}$ it is still as high as $94 \%$. The second and third columns of Fig. 1 show the NFIP at the 

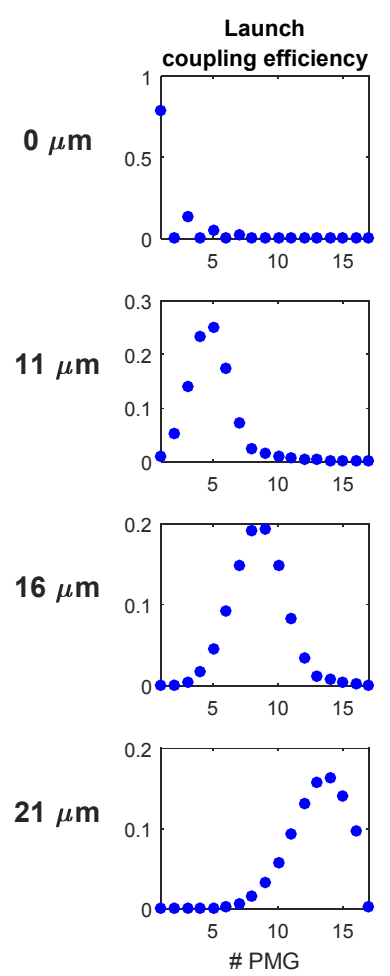
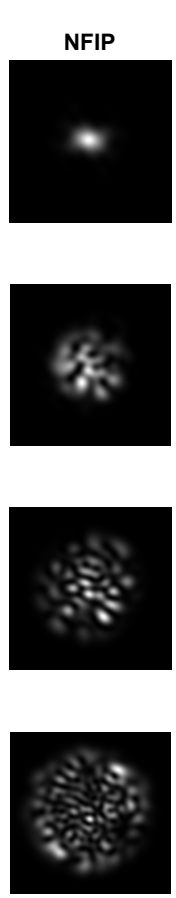
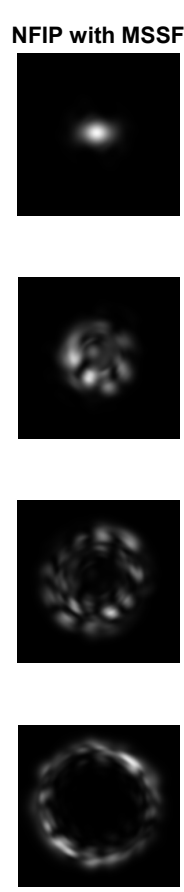
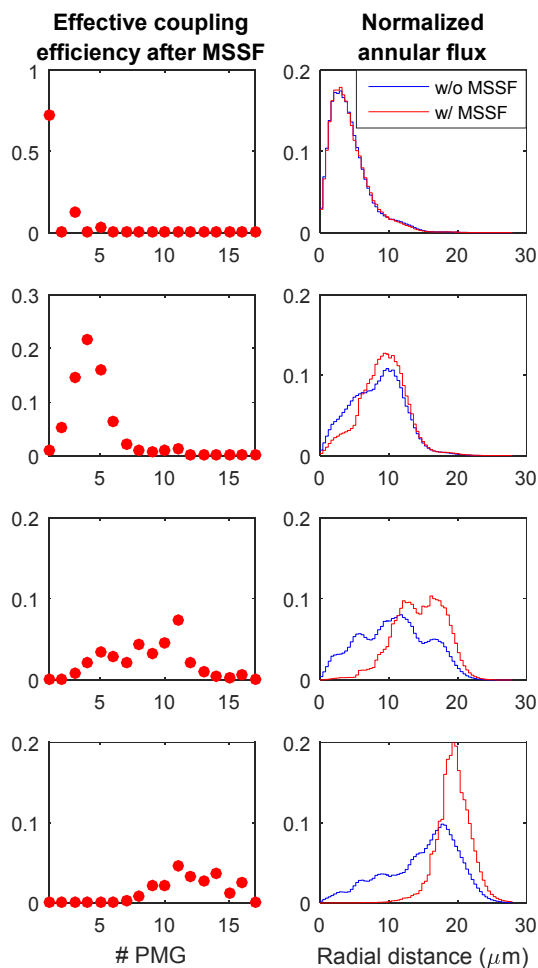

Figure 2. Similar as Fig. 1 for $940 \mathrm{~nm}$ wavelength.

output of the GI-MMF after $200 \mathrm{~m}$ of propagation, without and with MSSF, respectively. Full intra-PMG mixing is assumed. The power loss due to MSSF is $0.1,0.4,2.4$, and $4.5 \mathrm{~dB}$ for the four offset positions of $0,11,16$, and $21 \mu \mathrm{m}$, respectively. The effective coupling efficiency per PMG that corresponds to the NFIP with MSSF, i.e. the coupling efficiency per PMG that would arise if the near-field pattern with MSSF was used as the launch field into the GI-MMF, is shown in the fourth column of Fig. 1. Finally, the fifth column of Fig. 1 shows the normalized annular flux (NAF), calculated by integrating the near-field intensity over the azimuth angle from 0 to $2 \pi$ within radii $r$ and $r+d r$, with $d r=0.4 \mu \mathrm{m}$ [6]. The NAF shows the distribution of the NFIP along the radial dimension. Normalization is such that each NAF curve integrates to unity, in other words the coupling (launch and MSSF) and propagation loss is not depicted, since the objective is to show how the intensity is distributed along the radial dimension. The NAF is shown for both NFIPs, without and with MSSF. MSSF is clearly effective for the cases of 16 and $21 \mu \mathrm{m}$ offset, causing the NFIP to take a ring-like shape. Fig. 2 shows the corresponding results for $940 \mathrm{~nm}$ wavelength, whereas similar results have been obtained for 880 and $910 \mathrm{~nm}$.

The NAF with MSSF is shown in Fig. 3 for the four offset positions and the four wavelengths considered, for GI-MMF lengths of 30 and $300 \mathrm{~m}$. For the 30-m long GI-MMF no mode mixing is included, whereas for the $300-\mathrm{m}$ long GI-MMF full intra-PMG mixing is assumed. The dependence of the NFIP speckles on the phase and amplitude of the contributing modes causes changes to the NAF curves, which, however, follow the same trend for the four SWDM wavelengths and the two GI-MMF lengths considered. The results present the worst-case scenario, since a non-zero linewidth source will yield smoother NFIPs (lower contrast in the speckle pattern) as the MMF length increases [4]. The NAF curves can be used for the design of a receiver with multiple annular segments [6]. Assuming a multi-segment receiver is used, the remaining-after MSSF-overlap among the NFIPs of the spatial channels causes crosstalk that needs to be mitigated with multiple-input multiple-output (MIMO) signal processing techniques [6]. In intra-data-centre connections, keeping the cost and power consumption low is very important. Therefore, the degree of signal processing involved is strongly related to the dictated cost and power consumption restrictions [12]. However, from Fig. 3, it becomes clear that using two IM/DD SDM channels that correspond to offsets 0 and $21 \mu \mathrm{m}$ can be applied in a straightforward way, since the NAF curves are very well separated with MSSF for all four wavelengths. This optical spatial separation means that it is possible to apply IM/DD SDM transmission with two spatial channels within the SWDM wavelength range, with no additional MIMO signal processing being required.

\section{CONCLUSIONS}

The application in IM/DD GI-MMF transmission systems of SDM using offset excitation and MSSF, in combination with SWDM has been explored by simulating the NFIP at the output of a 50/125 $\mu \mathrm{m}$ GI-MMF. For four radially offset positions $(0,11,16$ and $21 \mu \mathrm{m})$ of the SMF at the input of the GI-MMF, the annular flux of the NFIPs after MSSF at the output of the GI-MMF follows the same trend for the four SWDM wavelengths 

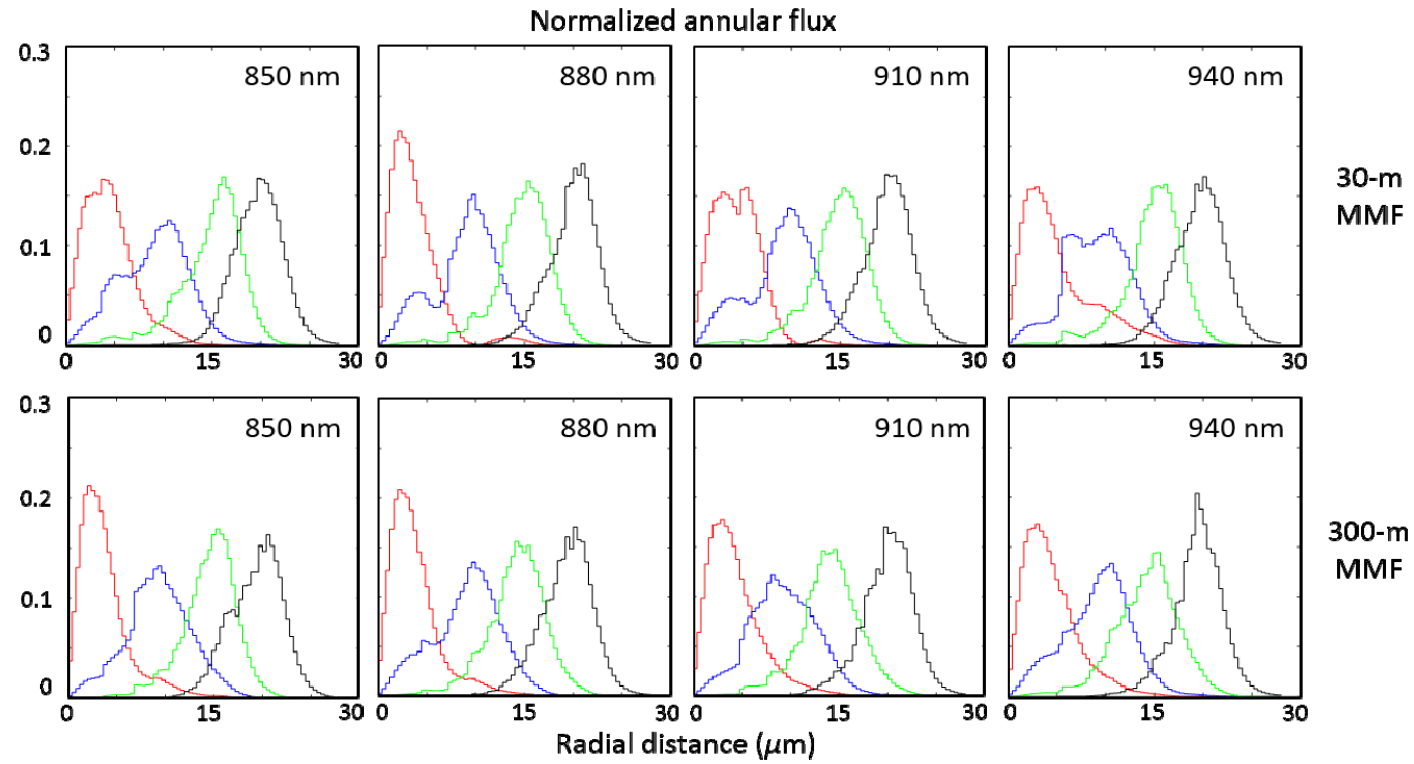

300-m MMF

Figure 3. Normalized annular flux with MSSF for 30-m of propagation without mode mixing (first row) and 300-m of propagation with full intra-PMG mixing (second row) at 850, 880, 910, and $940 \mathrm{~nm}$ wavelength. Each graph has four curves that correspond to an offset launch of 0 (red), 11 (blue), 16 (green), and 21 rm (black).

$(850,880,910$, and $940 \mathrm{~nm})$ considered. This means that common design specifications for a multi-segment receiving end can be drawn for this wavelength range [6]. Any residual spatial overlap among the NFIPs after MSSF gives rise to crosstalk among the spatial channels. Therefore, MIMO processing techniques are further required. However, the results show that it is possible to implement two IM/DD SDM channels-launched with offsets 0 and $21 \mu \mathrm{m}$ - across the SWDM wavelength range, separated by MSSF and not requiring additional MIMO signal processing.

\section{ACKNOWLEDGEMENTS}

This work has been partially supported by the EU FP7 project INSPACE (Grant 619732) and by the EPSRC project UNLOC (Grant EP/J017582/1).

\section{REFERENCES}

[1] R. Motaghian, et al:: 180 Gbps PAM4 VCSEL Transmission over 300m Wideband OM4 Fibre, in Proc. OFC 2016, Anaheim, California, USA, Mar. 2016, paper Th3G-2.

[2] Y. Sun, et al.: $51.56 \mathrm{~Gb} / \mathrm{s}$ SWDM PAM4 Transmission over Next Generation Wide Band Multimode Optical Fiber, in Proc. OFC 2016, Anaheim, California, USA, Mar. 2016, paper Tu2G-3.

[3] C. P. Tsekrekos, et al.: Mode-selective spatial filtering for increased robustness in a mode group diversity multiplexing link, Opt. Lett., vol. 32, no. 9, pp.1041-1043, May 2007.

[4] C. P. Tsekrekos, et al.: Mitigation of impairments in MGDM transmission with mode-selective spatial filtering, Photon. Technol. Lett., vol. 20, no. 13, pp.1112-1114, July 2008.

[5] T. Honda, et al.: Diffraction-compensated free-space WDM add-drop module with thin-film filters, Photon. Technol. Lett., vol. 15, no. 1, pp.69-71, Jan. 2003.

[6] C. P. Tsekrekos, et al.: Design considerations for a transparent mode group diversity multiplexing link, Photon. Technol. Lett., vol. 18, no. 22, pp. 2359-2361, Nov. 2006.

[7] M. Bigot, et al.: Extra-Wide-Band OM4 MMF for Future 1.6 Tbps Data Communications, in Proc. OFC 2015, Los Angeles, California, USA, Mar. 2015, paper M2C-4.

[8] I. H. Malitson: Interspecimen Comparison of the Refractive Index of Fused Silica, J. Opt. Soc. Am., vol. 55, no. 10, pp. 1205-1209, Oct. 1965.

[9] G. Yabre, et al.: Comprehensive Theory of Dispersion in Graded-Index Optical Fibers, J. Lightwave Technol., vol. 18, no. 2, pp. 166-177, Feb. 2000.

[10] J. Saijonmaa, et al.: Selective excitation of parabolic-index optical fibers by Gaussian beams, Appl. Opt., vol. 19, no. 14, pp. 2442-2452, July 1980.

[11] S. Kawakami, et al.: Evolution of power distribution in graded-index fibres. Elect. Lett., vol. 19, no. 3, pp. 100-102, Feb. 1983.

[12] M. Kuschnerov, et al.: Energy efficient digital signal processing, in Proc. OFC 2014, San Francisco, California, USA, Mar. 2014, paper Th3E-7. 